\title{
Impaired Fear Memory Specificity Associated with Deficient Endocannabinoid-Dependent Long-Term Plasticity
}

\author{
Jonathan W Lovelace', Philip A Vieira', Alex Corches ${ }^{2}$, Ken Mackie ${ }^{3}$ and Edward Korzus*, 1,2 \\ 'Department of Psychology and Neuroscience Program, University of California, Riverside, CA, USA; 'Biomedical Sciences Program, University \\ of California, Riverside, CA, USA; ${ }^{3}$ Department of Psychological and Brain Sciences, Gill Center for Biomedical Sciences, Indiana University, \\ Bloomington, IN, USA
}

\begin{abstract}
In addition to its central role in learning and memory, N-methyl D-aspartate receptor (NMDAR)-dependent signaling regulates central glutamatergic synapse maturation and has been implicated in schizophrenia. We have transiently induced NMDAR hypofunction in infant mice during postnatal days 7-I I, followed by testing fear memory specificity and presynaptic plasticity in the prefrontal cortex (PFC) in adult mice. We show that transient NMDAR hypofunction during early brain development, coinciding with the maturation of cortical plasticity results in a loss of an endocannabinoid (eCB)-mediated form of long-term depression (eCB-LTD) at adult central glutamatergic synapses, while another form of presynaptic long-term depression mediated by the metabotropic glutamate receptor 2/3 (mGluR2/ 3-LTD) remains intact. Mice with this selective impairment of presynaptic plasticity also showed deficits in fear memory specificity. The observed deficit in cortical presynaptic plasticity may represent a neural maladaptation contributing to network instability and abnormal cognitive functioning.

Neuropsychopharmacology (2014) 39, 1685-1693; doi:10.1038/npp.2014.15; published online 19 February 2014
\end{abstract}

Keywords: NMDA receptor; endocannabinoid system; memory specificity; pattern separation; synaptic plasticity; schizophrenia

\section{INTRODUCTION}

The ability to discriminate between similar, yet different, contexts is critical for episodic memory (Tulving, 2002). Episodic memory is demonstrably abnormal in schizophrenia (Pelletier et al, 2005) and imbalanced memory specificity and generality may occur independent of associative learning in schizophrenia patients (Tamminga, 2013). Although studies on context discrimination have focused on the hippocampal circuit (Leutgeb et al, 2007; McHugh et al, 2007; Sahay et al, 2011), recent studies have demonstrated that the neural circuit for memory specificity and generalization comprises an anatomically widespread circuit including the medial PFC (mPFC) (Navawongse and Eichenbaum, 2013; Xu and Sudhof, 2013).

Clinical data and neuroimaging studies indicate that schizophrenia-specific cognitive deficits related to prefrontal circuit pathophysiology are present before the onset of psychosis (Reichenberg et al, 2010). In addition, there is converging evidence linking the schizophrenia cognitive endophenotype with $\mathrm{N}$-methyl D-aspartate receptor (NMDAR) hypofunction in the corticolimbic system (Coyle et al, 2003; Tamminga, 1998). Exposure to

\footnotetext{
*Correspondence: Dr E Korzus, Department of Psychology and Neuroscience Program, University of California Riverside, 900 University Avenue, Riverside, CA 92521, USA, Tel: + I 951 827 4992, Fax: + I 95 I 827 3985, E-mail: edkorzus@ucr.edu Received 30 August 2013; revised 20 January 2014; accepted 21 January 20I4; accepted article preview online 24 January 2014
}

dissociative anesthetics such as phencyclidine (PCP) or ketamine, which are NMDAR antagonists, produces schizophrenia-like symptoms (Javitt and Zukin, 1991), and acute, chronic, or perinatal treatment with NMDAR antagonists produce cognitive deficits similar to schizophrenia-like phenotypes in rodents (Morris et al, 2005).

Although the idea of deficient memory specificity and overgenerality in psychosis is appealing, there has been no experimental data to support this hypothesis. Conversely, the fundamental question regarding how NMDARs function to sculpt the capacity for presynaptic forms of plasticity during early postnatal development has not been elucidated. To address these questions, we examined fear memory specificity and presynaptic forms of cortical plasticity in the mPFC in an adult mouse model with induced transient PCP-mediated NMDAR hypofunction during early brain development coinciding with the maturation of cortical plasticity. PCP-treated mice exhibited impairment in contextual discrimination tasks, indicating deficient fear memory specificity. We also examined multiple forms of presynaptic plasticity at the cortical layer $2 / 3$ to 5 (L2/3 $\rightarrow$ L5) glutamatergic synapse in the mPFC in PCP-treated mice. Unexpectedly, PCP-treated mice showed a strong deficit in eCB-LTD in the MPFC, whereas mGluR2/3-LTD and multiple forms of presynaptic short-term plasticity were spared. Thus, the maturation of presynaptic long-term plasticity at this cortical glutamatergic synapse is sensitive to NMDAR hypofunction during early postnatal development. The observed loss of eCB-LTD may result from a 
maladaptation in the excitatory circuitry, which is associated with deficient cognitive functioning.

\section{MATERIALS AND METHODS}

\section{Subjects}

C57BL/6 mice were used for all of the experiments following protocols approved by the IACUC at UCR. The animals were housed in plastic cages (2-4 mice per cage) and kept on $12 / 12 \mathrm{~h}$ dark/light cycle with ad libitum access to food and water. All of the behavioral experiments were performed during the light phase of the cycle. Ten- to seventeen-weekold mice were used for the physiological and behavioral studies. To generate the PCP-treated animals, C57BL/6 mice are subcutaneously injected with a $10 \mathrm{mg} / \mathrm{kg}$ per day dose of PCP-HCl (Sigma) at the same time of the day on postnatal days (P) 7, 9, and 11 as described previously (Wang et al, 2001). All of the behavioral and physiological tests were performed using equal gender distribution (males/females, 50\%/50\%) during adulthood (P70-120).

\section{Behavioral Assays}

Fear conditioning is performed as described before (Korzus et al, 2004). After being handled, individual mice were exposed to a fear conditioning box (Coulburn Instruments), which was placed inside of a sound-attenuated chamber with the house light and house fan on. The chamber was cleaned with Quatracide, $70 \%$ ethanol, and distilled water. The individual mice were placed for $180 \mathrm{~s}$ and received a $0.75 \mathrm{~mA}, 2 \mathrm{~s}$ footshock (context-footshock pairing), and left for another $180 \mathrm{~s}$ inside the chamber. For memory retention test, mice are placed back in the training chamber for $180 \mathrm{~s}$. Freezing was scored and analyzed automatically by a videobased system (Freeze Frame software; ActiMetrics). Video was recorded at 30 frames/s. The Freeze Frame software calculated the difference between consecutive frames by comparing grayscale value for each pixel in frame. Freezing was defined based on experimenter observations and set as subthreshold activity for longer than $1 \mathrm{~s}$. Freezing was expressed as \% Freezing, which was calculated as the percent of freezing time per total time spent in the testing chamber. Contextual fear conditioning and a 24-h memory test (Figure 1a; Days 1 and 2) were followed by context discrimination assay (Figure 1a, Days $2-12)$. The context discrimination assay was performed similarly as described before (McHugh et al, 2007). The task was divided into three phases: initial phase, generalization phase, and discrimination phase. After being handled, individual mice were exposed to context $\mathrm{A}$. Context $\mathrm{A}$ was the unmodified fear conditioning box (Coulburn Instruments). The chamber was cleaned with Quatricide, 70\% ethanol, and distilled water. Context B was the modified fear conditioning chamber, with angular wall inserts, house fan off, and scented with Simple Green. During the initial phase (Days 1 and 2), mice were placed in the context A (CS +) for $180 \mathrm{~s}$ followed by a single footshock (arrow). Across 4 consecutive days (generalization phase: Days 3-6; Trial Blocks 1 and 2), the individual mice were exposed to context $\mathrm{A}$ for $180 \mathrm{~s}$ and received a $0.75 \mathrm{~mA}, 2 \mathrm{~s}$ footshock, and left for another $60 \mathrm{~s}$ inside the chamber. After $4 \mathrm{~h}$, the mice were exposed to the similar context B for $242 \mathrm{~s}$ and receive no footshock. During the generalization phase, mice were not able to discriminate between context A and context B. On Days 7-12 (discrimination phase: Trial Blocks 3-5), mice were placed again in the context A (CS + ) for $180 \mathrm{~s}$ followed by a single footshock, and left for $60 \mathrm{~s}$ after shock, and in the context B (CS - ) for $242 \mathrm{~s}$ without any reinforcement. CB57BLJ6 mice acquired the ability to discriminate between context $\mathrm{A}$ and context $\mathrm{B}$ during the discrimination phase after at least 6 days of training. Contexts A and B were similar but not the same. The protocol included 12 days of training. The order of exposure to different contexts was counterbalanced. In addition, the context cues themselves were counterbalanced within each group to isolate the effect of the CS + .

Additional detailed description of Materials and methods is included in Supplementary Materials.

\section{RESULTS}

\section{PCP-Treated Mice Exhibit Deficit in Fear Memory Specificity}

To investigate the developmental role of NMDARs on cognitive function in adulthood, we induced transient PCPmediated NMDAR hypofunction in perinatal mice on P7-11 (Wang et al, 2001), which overlaps with a period of extensive cortical plasticity maturation (Zhang, 2004). We found that PCP-treated mice performed similar to vehicle-treated controls (CTRL) in the contextual version of the fear conditioning task (Supplementary Figure S1a) and showed the same freezing after a $24 \mathrm{~h}$ delay (CTRL: $24.91 \pm 4.90 \%, n=19$; PCP treated: $25.09 \pm 5.18 \%, n=16 ; t_{(33)}=0.979, p=0.979$, $r=0.17)$. Next, we subjected the PCP-treated mice to a context discrimination task (McHugh et al, 2007) to evaluate the role of NMDARs during early postnatal development in the establishment of neural mechanisms underlying fear memory specificity (Figure 1a). PCP-treated and control mice exhibit comparable freezing to both contexts during the first trial block (Figure 1d; two-way analysis of variance (ANOVA) of context and treatment during trial block 1; Context $\times$ Treatment: $\mathrm{F}_{(1,66)}=0.06, p=0.810$ ). In fact, a similar level of freezing was observed in both groups during the entire generalization phase (see Figures $1 \mathrm{~b}$ and $\mathrm{c}$ ). This indicates that context A was similar enough to context B that strong generalization was observed during the generalization phase, and freezing in contexts A and B was comparable in both groups.

The control group began to freeze significantly less to context B compared with context A after training on context discrimination task, demonstrating the ability to distinguish consistently between similar yet different contexts (repeated measures (RM) ANOVA of trial blocks 1-5 and context: Context: $\mathrm{F}_{(1,18)}=23.33, p=0.0001$; Trial Block, $\mathrm{F}_{(4,72)}=4.74, p=0.002$; Trial Block $\times$ Context: $\mathrm{F}_{(4,72)}=4.70$, $p=0.002)$. Post hoc analysis using Bonferroni correction for multiple comparisons indicates that differences were present during the discrimination phase: trial blocks 4 $(p=0.0008)$ and $5(p=0.0002)$ only. In contrast to control animals, PCP-treated mice exhibit a deficit in fear memory specificity (Figure 1c; RM-ANOVA of trial blocks $1-5$ and context: Context: $\mathrm{F}_{(1,15)}=6.41, p=0.023$; Trial Block: $\mathrm{F}_{(4,60)}=0.86, p=0.49$; Trial Block $\times$ Context: $\mathrm{F}_{(4,60)}=0.79$, $p=0.535)$. 


\section{a}
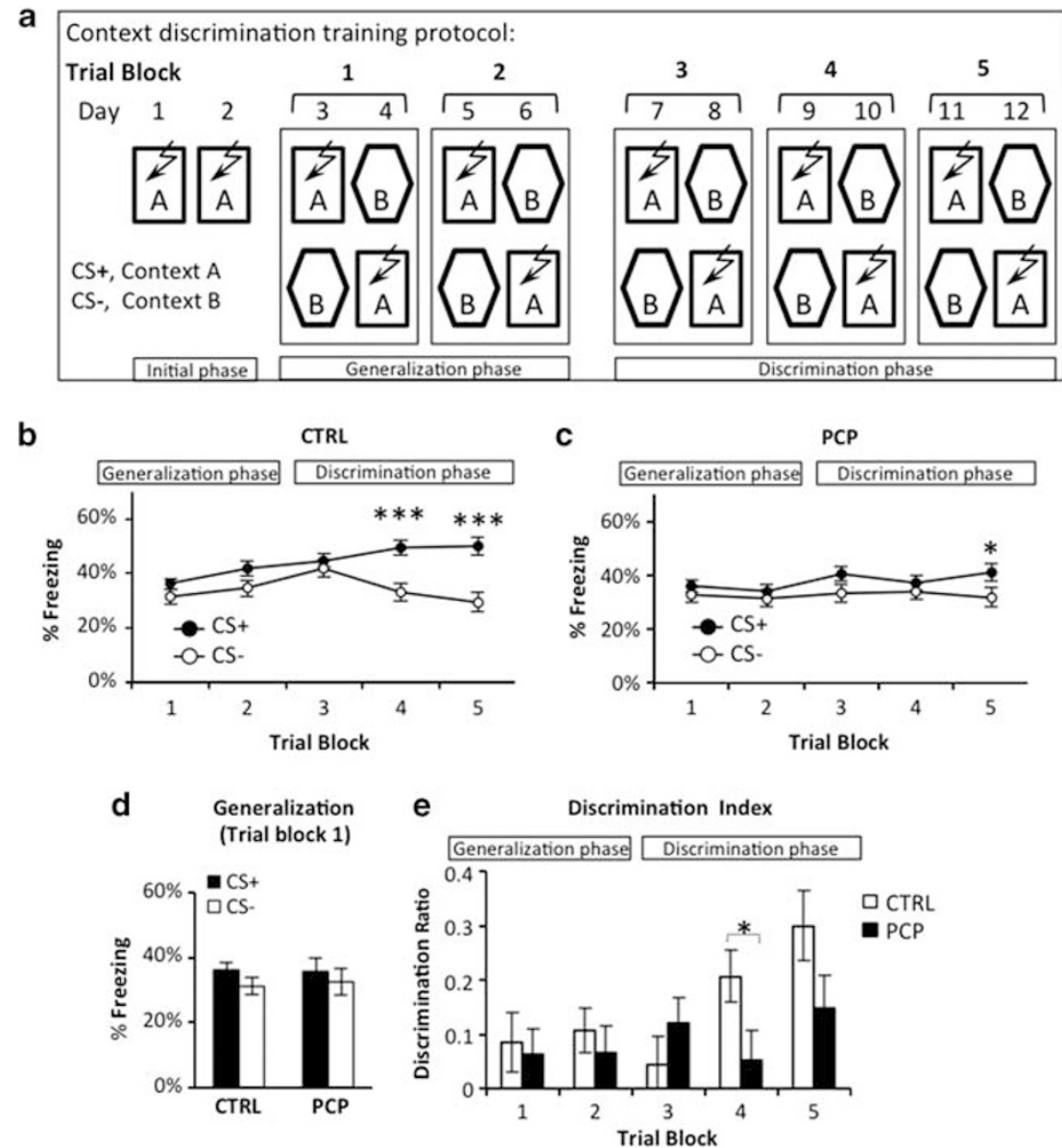

Figure I Fear memory specificity is deficient in phencyclidine (PCP)-treated mice. (a) Experimental design for the context discrimination test. The task is divided into three phases: initial phase, generalization phase, and discrimination phase. During initial phase (Days I and 2), mice were placed in the context A (CS +) for 180 s followed by a single footshock (arrow). On Days 3-6 (generalization phase: Trial Blocks I and 2), mice were placed in the context A $(\mathrm{CS}+)$ for $180 \mathrm{~s}$ followed by a single footshock (arrow), and left for $60 \mathrm{~s}$ after shock, and in context B (CS - ) for $242 \mathrm{~s}$ without any reinforcement. On Days 7-12 (discrimination phase: Trial Blocks 3-5), mice were placed again in the context A (CS + ) for $180 \mathrm{~s}$ followed by a single footshock (arrow), and left for $60 \mathrm{~s}$ after shock, and in context B (CS - ) for $242 \mathrm{~s}$ without any reinforcement. Contexts A and B were similar but not the same. The protocol included 12 days of training. (b) During the generalization phase, CB57BL/16 control mice $(n=19)$ were not able to discriminate between context $A$ and context $B$ (repeated measures-analysis of variance (RM-ANOVA): Trial Block $\times$ Context: $\left.F_{(1,18)}=0.356, p=0.558\right)$. However, CB57BL/J6 control mice acquired the ability to discriminate between context $\mathrm{A}$ and context $\mathrm{B}$ they discriminate after 8 days, according to the graph (RM-ANOVA: Trial Block $\times$ Context: $\left.F_{(2.36)}=5.728, p=0.007\right)$. (c) PCP-treated mice $(n=16)$ exhibit deficit in fear memory specificity during the discrimination phase (RM-ANOVA: Trial Block $\times$ Context: $\left.F_{(2,30)}=0.895, p=0.419\right)$. (d) Freezing in both tested groups had comparable levels to both contexts during the first block. This would indicate that context $A$ was similar enough to context $B$ that generalization occurred early in training. (e) Analysis of context discrimination ratios (DI) were calculated according to the formula: $\mathrm{DI}=(($ context $\mathrm{A}$ - context $\mathrm{B}) /($ context $\mathrm{A}+$ context $\mathrm{B}))$ revealed difference in performance between $\mathrm{PCP}$-treated and control mice during the discrimination phase (trial block 4). Asterisks indicate statistical significance, $* p<0.05, * * p<0.01, * * * p<0.001$.

PCP-treated animals demonstrated a strong deficit in memory specificity during the discrimination phase when compared with controls (Figures $1 b$ and c; RM-ANOVA: Treatment $\times$ Context $\times$ Trial Blocks 3-5: $\quad \mathrm{F}_{(2,66)}=3.411$, $p=0.039)$. Control mice acquired the ability to distinguish contexts A and B during trial block 4, $t_{(18)}=4.026$, $p=0.0008, r=0.69$ and also showed strong fear memory specificity during trial block $5, t_{(18)}=4.754, p=0.0002$, $r=0.75$. Contrary to the control, PCP-treated mice were not able to discriminate contexts $\mathrm{A}$ and $\mathrm{B}$ on trial block 4 (a two-way ANOVA of treatment and context on trial block 4: Context $\times$ Treatment: $\mathrm{F}_{(1,66)}=4.64, p=0.035$; PCP treated: $\left.t_{(15)}=0.776, p=0.450, r=0.20\right)$. During the last trial block (Figure 1c), PCP-treated mice slightly improved their ability to discriminate contexts A and B (PCP treated: $t_{(15)}=2.429$, $p=0.028, r=0.53$ ), which suggests that PCP-treated mice overcame their context discrimination deficit with addi- tional training compared with controls (a two-way ANOVA of treatment and context on trial block 5: Context $\times$ Treatment: $\left.\mathrm{F}_{(1,66)}=2.88, p=0.094\right)$. To clarify this effect, we have also compared context discrimination ratios between groups during trial blocks. Figure $1 \mathrm{e}$ shows a marked difference on trial block $4\left(t_{(33)}=2.121, p=0.042, r=0.74\right)$, but no difference between groups on trial block 5 $\left(t_{(33)}=1.670, p=0.10, r=0.28\right)$, indicating that as training continues, PCP-treated animals' performance becomes closer to control animals' performance. In summary, these data strongly suggest that transient NMDAR hypofunction during early postnatal development (P7-11) resulted in deficient fear memory specificity in the adult.

In addition, PCP-treated mice showed normal levels of uninduced locomotor activity in a novel environment (Supplementary Figures S1d-f; Average Velocity: PCP treated: $4.85 \pm 0.15 \mathrm{~cm} / \mathrm{s}, n=33$; CTRL: $5.07 \pm 0.20 \mathrm{~cm} / \mathrm{s}, n=30$; 
$t_{(61)}=0.859, p=0.3939, r=0.11$; Total Distance Traveled: PCP treated: $43.42 \pm 1.28 \mathrm{~m}, n=33$; CTRL: $45.56 \pm 1.77 \mathrm{~m}$, $\left.n=30 ; t_{(61)}=0.998, \quad p=0.3262, \quad r=0.13\right)$ and normal anxiety-related responses expressed as \% time spent in the center of the open field (PCP treated: $29.95 \pm 0.98 \%$, $n=33$; CTRL: $26.48 \pm 1.84 \%, \quad n=30 ; \quad t_{(61)}=1.709, \quad p=$ $0.0926, r=0.21$ ) (Supplementary Figure S1f), which is consistent with the previous study (Nakatani-Pawlak et al, 2009). Consistent with a previous report (Grayson et al, 2007; Nakatani-Pawlak et al, 2009; Wiley et al, 2003), PCPtreated mice exhibited abnormal behavior in the novel object recognition task (Supplementary Figures S3a and b) but normal performance in the object location task (Supplementary Figures S3c-f). One difference between these two behavioral tasks is that novel object recognition memory test involves exposure to novelty (which may generate mild fear), while object location test relies entirely on familiar context and objects.

\section{mGluR2/3-Dependent LTD is Unaffected in PCP-Treated Mice}

Convergent cortical and subcortical pathways are integrated in cortical L2/3 pyramidal neurons, which form abundant contacts with pyramidal neurons in cortical L5, the output of the cortex. To investigate the developmental role of NMDA receptors in the maturation of presynaptic plasticity, we tested synaptic activity at the L2/3 $\rightarrow$ L5 glutamatergic synapse (Hempel et al, 2000; Morris et al, 1999) (Figures 2 and 3 and Supplementary Figure S2). One way to capture how a synapse transforms signals is to analyze relation between its input and output (I/O). Analysis of the shape of the I/O curve may provide valuable information about additive operations (shift along the input axis) or gain (slope change). I/O relations appear to be the same in PCPtreated and control mice (Supplementary Figure S5b). Additional detail analysis of I/O relations was carried out to determine if differences in the shape of the curves existed using different metrics obtained from Boltzmann sigmoidal analysis. Comparing means of individual Boltzmann fitted parameters revealed no differences between PCP-treated and control mice in maximum asymptote (A2: PCP treated: $0.68 \pm 0.057 \mathrm{mV}, n=11$; CTRL: $0.78 \pm 0.06 \mathrm{mV}, n=9$; $t$-test: $p>0.05$ ), in the center ( $\mathrm{x} 0$ : PCP treated: $79.46 \pm 9.98 \mu \mathrm{A}$, $n=11$; CTRL: $81.63 \pm 10.34 \mu \mathrm{A}, n=9 ; p>0.05)$, or in the time constant (dx: PCP treated: $22.36 \pm 3.67, n=11$; CTRL: $27.63 \pm 4.53, n=9$; $t$-test: $p>0.05)$. This suggests that there are no differences in all tested characteristics of the I/O curves. Thus, by these measures, PCP-treated mice showed unaltered synaptic transmission in the L2/3 $\rightarrow$ L5 pathway, indicating normal synaptic density within the population, and that synaptic transmission was equally effective in response to a single stimulus.

mGluR2/3 receptors' activation is known to induce LTD (mGluR2/3-LTD) with a presynaptic locus of expression in the PFC (Robbe et al, 2002). We tested mGluR2/3-LTD in $\mathrm{PCP}$-treated mice in the L $2 / 3 \rightarrow \mathrm{L} 5$ pathway. Success or failure of LTD expression in individual groups was determined using a paired Wilcoxon's signed rank test (Wilcoxon's test) by comparing the mean fPSP amplitude during baseline (10 min before stimulation) and 50-60 min after LTD induction. Figure 2 shows that the mGluR2/3 agonist
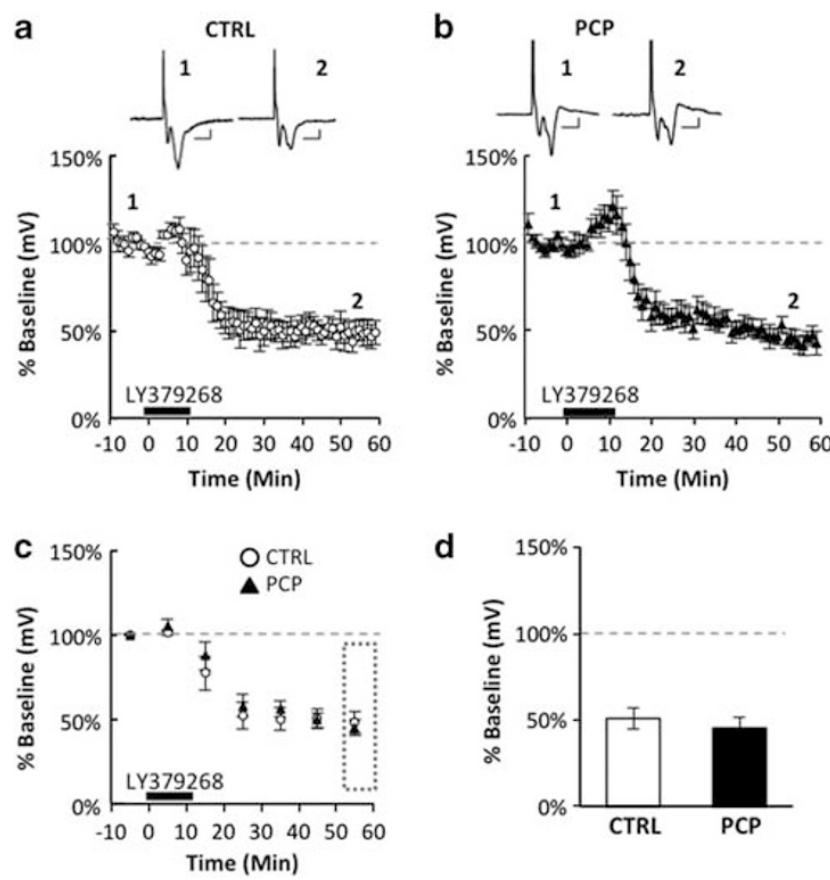

Figure 2 Metabotropic glutamate receptor 2/3-dependent long-term depression (mGluR2/3-LTD) at the layer $(\mathrm{L}) 2 / 3 \rightarrow \mathrm{L} 5$ synapse is unaffected in phencyclidine (PCP)-treated mice. mGluR2/3-LTD was induced at time 0 with 10 min bath application of 100 nM LY379 268 (potent mGluR2/3 agonist) in the $L 2 / 3 \rightarrow L 5$ pathway. Example traces are shown for each group corresponding to respective time points. The CTRL group (a) showed significant expression of LTD as well as the PCP-treated group (b), suggesting that mGluR2/3-dependent LTD is unaffected in PCP-treated mice. Insets: Traces shown indicate representative average from $10 \mathrm{~min}$ of baseline ('I') and the time between 50 and 60 min of recording ('2') (scale bar: vertical $=0.1 \mathrm{mV}$; horizontal $=5 \mathrm{~ms}$ ). (c) Binned averages of every 10 min across time show similarity in the mGluR2/3-LTD expression levels in the PCP-treated and control (CTRL) animals at the $L 2 / 3 \rightarrow L 5$ synapse. (d) There is no difference in the expression of mGLuR2/3-LTD between CTRL and PCP-treated mice after $60 \mathrm{~min}$ (CTRL: $50.82 \pm 6.03 \%, n=7$; PCP treated: $45.31 \pm 4.41 \%, n=7 ; t_{(12)}=0.738, p=0.47, r=0.21$ ).

LY379 268 was effective in inducing robust mGluR2/3-LTD in the control and PCP-treated mice (Wilcoxon's test: CTRL: $50.83 \pm 6.03 \%, Z=-2.366, n=7, p=0.018$; PCP treated: $45.31 \pm 4.41 \%, Z=-2.366, n=7, p=0.018)$. There was no difference between the control and PCP-treated mice in the level of mGluR2/3-LTD expression (Figure 2c; RM-ANOVA: Time $\times$ Treatment: $\left.F_{(2,24)}=1.125, p=0.305\right)$, indicating that mechanisms controlling presynaptic mGluR2/3-LTD, including those shared with eCB-LTD (see below), are intact in PCP-treated mice. In addition, PCP-treated mice show normal short-term forms of presynaptic plasticity at the L2/3 $\rightarrow$ L5 glutamatergic synapse (Supplementary Figure S5).

\section{PCP-Treated Mice Show Deficient eCB-Dependent Cortical Plasticity}

eCB-LTD is a widespread form of cortical plasticity that provides activity-dependent inhibitory control of neurotransmitter release. The cannabinoid receptor type 1 (CB1R) is required for the induction of eCB-LTD (Lovinger, 2008). Similar to mGluR2/3-LTD, eCB-LTD is also a presynaptic form of plasticity that is negatively coupled to the CAMP/PKA signaling pathway via Gi/o (Lovinger, 2008). Thus, we 

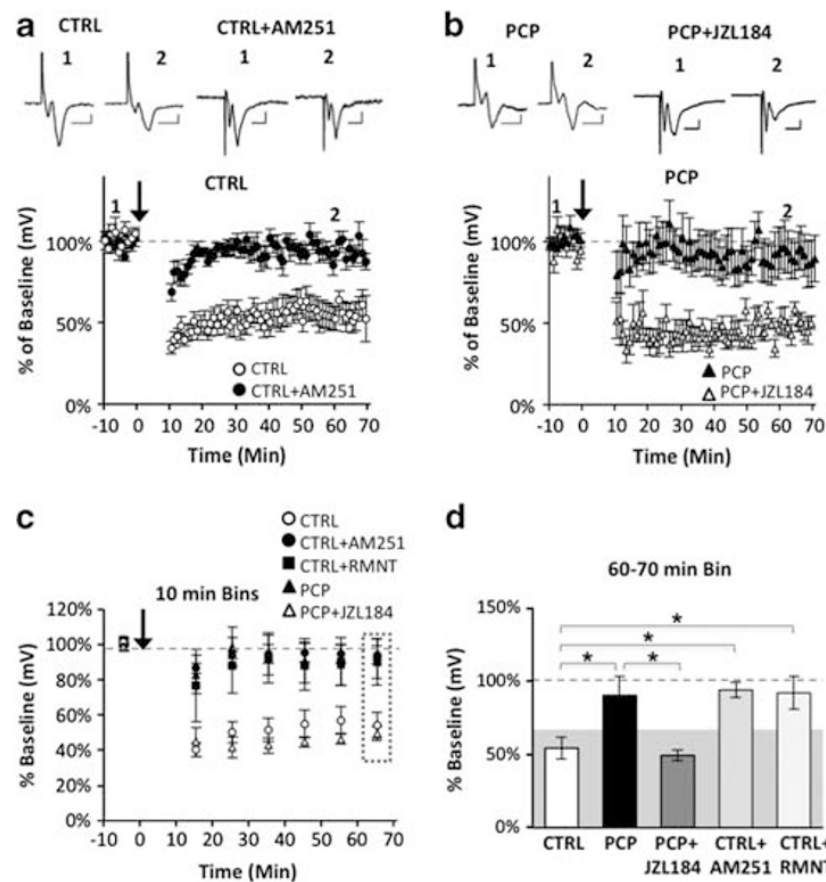

d

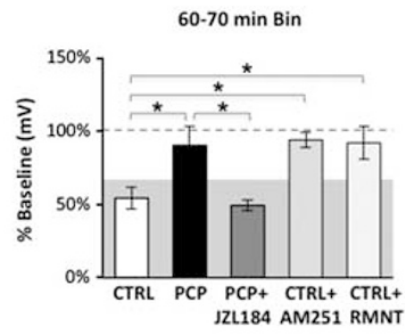

Figure 3 Endocannabinoid (eCB)-dependent long-term depression (eCB-LTD) at the layer (L)2/3 $\rightarrow$ L5 synapse is deficient in phencyclidine (PCP)-treated mice. (a) The control (CTRL) group showed robust expression of eCB-LTD at the $L 2 / 3 \rightarrow L 5$ synapse in the medial prefrontal cortex (mPFC). eCB-LTD was induced at time 0 (black arrows) with a $10 \mathrm{~Hz}$ train applied for $10 \mathrm{~min}$. LTD magnitude was estimated from field postsynaptic potentials (IPSPs) registered during the period of 60-70 min (2) after LTD induction as a percentage of baseline fPSP amplitudes. Bath application of $5 \mu \mathrm{M}$ AM25 I completely blocked LTD, indicating that this is an eCB-dependent form of LTD. Insets: Traces shown indicate representative average from $10 \mathrm{~min}$ of baseline (' $\mathrm{I}$ ') and the time between 60 and 70 min of recording (' 2 ') (scale bar: vertical $=0.1 \mathrm{mV}$; horizontal $=5$ $\mathrm{ms}$ ). (b) The PCP-treated group showed no induction of LTD at the $L 2 / 3 \rightarrow L 5$ synapse in the $\mathrm{mPFC}$. However, stimulation of $10 \mathrm{~Hz}$ for 10 min to PCP slices in the presence of JZLI84 was able to rescue the deficit (Wilcoxon's test: PCP-treated +JZLI84: $49.38 \pm 3.78 \%, \quad Z=-2.366$, $n=7, p=0.018$ ). (c) Binned averages of every 10 min show distinct differences between all experimental conditions. (d) Data are plotted from the binned average time point in the rectangle marked during the last 10 min of recording. Control recordings show the expression of eCB-LTD, whereas PCP-treated slices and control slices with bath application of AM25 I or Rmnt showed no induction of eCB-LTD, and JZLI 84 application to PCP slices brought the level of LTD down to control levels. Asterisks indicate statistical significance, $* p<0.05$, $* * * 0.01$, **** $p<0.001$.

examined eCB-LTD in PCP-treated mice in the L2/3 $\rightarrow \mathrm{L} 5$ pathway. We used a moderate stimulation protocol of $10 \mathrm{~Hz}$ for $10 \mathrm{~min}$ at $70 \%$ stimulus intensity to induce eCB-LTD at the L2/3 $\rightarrow$ L5 glutamatergic synapse in the MPFC (Figure 3a), which has been shown to produce long-term depressive effects lasting for at least $1 \mathrm{~h}$ (Lafourcade et al, 2007). A comparative analysis of the mean fPSP amplitude during baseline and 60-70 min after LTD induction revealed robust LTD expression in control animals (Figure 3a; Wilcoxon's test: $54.30 \pm 7.34 \%, Z=-2.521 n=8, p=0.012$ ). It is well established that the expression of this form of LTD is coupled with an $\mathrm{eCB}$ receptor (CB1R) (Lafourcade et al, 2007; Lovinger, 2008). Not surprisingly, bath application of CB1R inhibitors, such as AM251 (Figure 3a) or SR141716 (Rimonabant or Rmnt) (Supplementary Figure S4d), blocked the induction of eCB-LTD in acute brain slices isolated from control animals in the L2/3 $\rightarrow$ L5 pathway (Figure 3c; Wilcoxon's test: AM251: $94.26 \pm 5.25 \%, Z=-0.734, n=6$, $p=0.463 ; \quad$ Rmnt: $92.05 \pm 11.41 \%, \quad Z=-0.813, \quad n=5$, $p=0.416$ ). Comparative analysis of the last $10 \mathrm{~min}$ of recordings (Figure 3d) reveals difference between control and AM251- or Rmnt-treated slices (AM251: 94.26 5 5.25\%, $n=6 ; \quad$ CTRL: $\quad 54.30 \pm 7.34 \%, \quad n=8 ; \quad$ AM251 vs CTRL: $t_{(12)}=-4.134, p=0.00138, r=0.77$; Rmnt: $92.05 \pm 11.41 \%$, $n=5$; Rmnt $v s$ CTRL: $\left.t_{(11)}=-2.929, p=0.0137, r=0.66\right)$.

Unexpectedly, the PCP-treated mice showed a strong deficit in eCB-LTD induction in the L2/3 $\rightarrow$ L5 pathway in the $\mathrm{mPFC}$ (Figure 3b; Wilcoxon's test: $90.29 \pm 12.6 \%$, $Z=-0.866, n=10, p=0.386)$. In contrast to the control animals, where the L2/3 $\rightarrow$ L5 pathway showed a robust and reproducible expression of the eCB-LTD in response to the $10 \mathrm{~Hz}$ stimulus, the recordings in the PCP-treated mice showed strong deficit in eCB-LTD expression in response to the $10 \mathrm{~Hz}$ stimulation protocol (Figure 3e; CTRL: $54.30 \pm 7.34 \%, n=8$; PCP treated: $90.29 \pm 12.6 \%, n=10$; $\left.t_{(16)}=2.211, p=0.0420, r=0.48\right)$. Blockade of the monoacylglycerol lipase (MAGL), the primary enzyme responsible for degrading the eCB 2-arachidonoylglycerol (Makara et al, 2005), with the specific inhibitor JZL184 (Long et al, 2009) reversed eCB-LTD deficit found in the PCP-treated mice. (Figure 3b; Wilcoxon's test for PCP-treated + JZL184: $49.38 \pm$ $3.78 \%, Z=-2.366, n=7, p=0.018$; PCP treated $v s$ PCP treated + JZL184: $\quad 49.38 \pm 3.78 \%, \quad n=7 ; \quad t_{(15)}=2.509$, $p=0.024, r=0.54)$. Altogether, these experiments strongly suggest that the $\mathrm{eCB}$ system is disrupted in PCP-treated mice.

\section{CB1R Signaling in the MPFC is Reduced in PCP-Treated Mice}

Induction of eCB-LTD can be effectively abolished via the direct inhibition of CB1R function (Figure 3c), which is consistent with previous reports (Lovinger, 2008). It is reasonable to assume that the observed deficits in eCB-LTD could result from the development of abnormal CB1Rdependent signaling in PCP-treated mice as a compensatory mechanism of the mPFC network to the abnormal maturation of a glutamatergic synapse. Thus, we examined CB1R expression in PCP-treated mice using immunohistochemistry (Figures $4 \mathrm{a}$ and $\mathrm{b}$ ). The PCP-treated mice showed a modest decrease in CB1R in the mPFC (PCP treated: $0.81 \pm 0.037, n=33$; CTRL: $1 \pm 0.036, n=23 ; t_{(54)}=3.605$, $p=0.0007, r=0.44)$. Next, we measured the direct responsiveness of CB1Rs to its well-characterized agonist Win55212-2 using an acute brain slice preparation in PCP-treated and control animals. Evoked fPSPs in L5 stimulated at the L2/3 inputs in the MPFC were strongly inhibited by a bath perfusion of the $1 \mu \mathrm{M}$ Win55212-2 in control animals (Figure 5), while the PCP-treated mice showed a modest reduction of depressed responses (Figure 5a; RM-ANOVA: Time $\times$ Treatment: $\mathrm{F}_{(2,27)}=8.27$, $p=0.000003)$, which correlated with a decrease in CB1R immunoreactivity (Figure 4a). The size of PPRs measured before and during $1 \mu \mathrm{M}$ Win55212-2 bath application corresponded to the level of recorded depression of fPSPs (Figure 5b). This increase in PPR is consistent with a presynaptic locus of action. The observed decrease in CB1R function is consistent with the observed loss of eCB-LTD (Figure $3 \mathrm{~b}$ ) and provides direct evidence for deficient eCB 


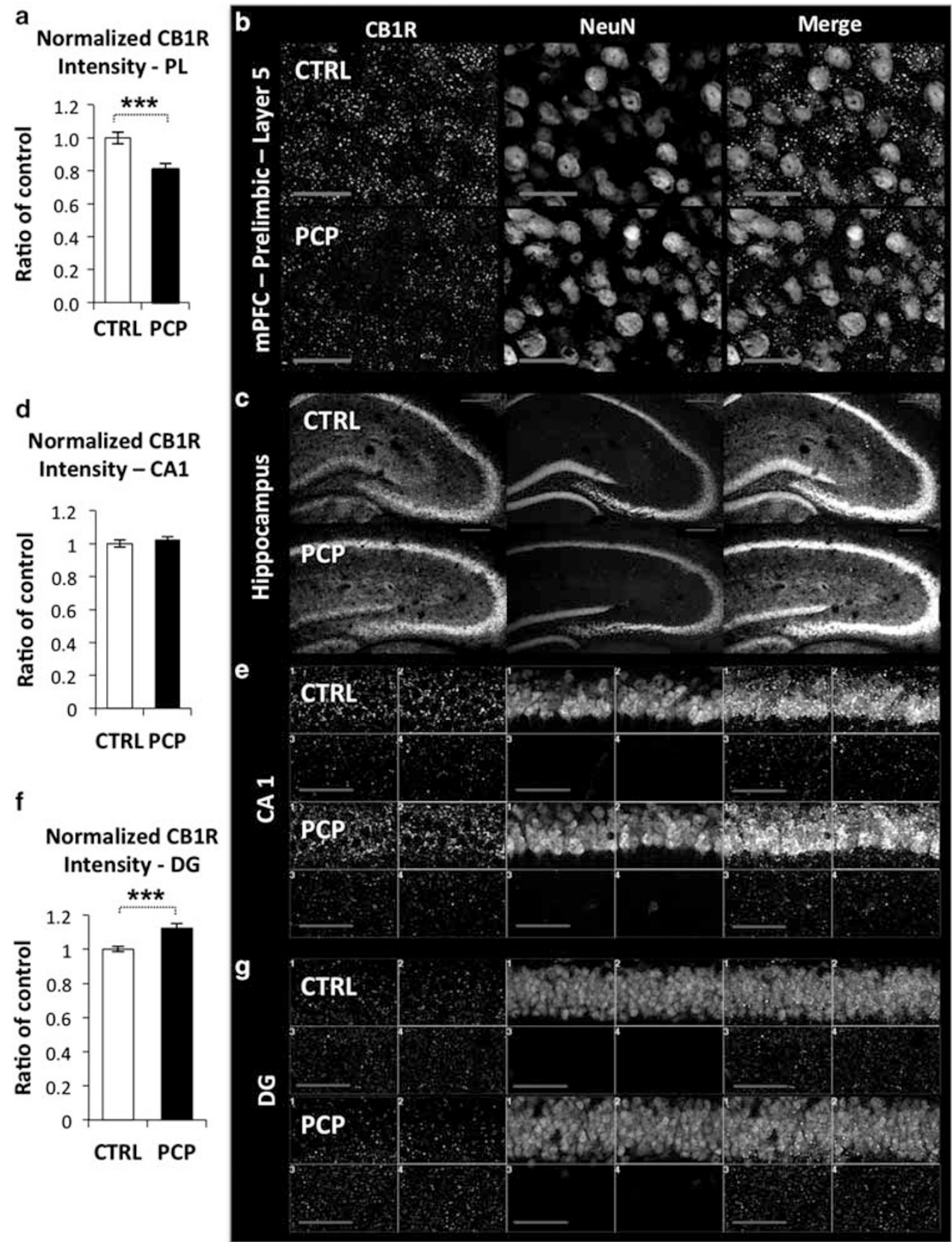

Figure 4 Cannabinoid receptor type I (CBIR) expression is reduced in phencyclidine (PCP)-treated mice. (a) Mean intensity of the CBIR fluorescent signal (normalized to NeuN signal) in layer 5 of the prelimbic-medial prefrontal cortex (PL-mPFC) of PCP-treated mice showed a marked decrease when normalized to the control (CTRL) group (PCP treated: $0.81 \pm 0.037, n=33$ slices ( 3 animals); CTRL: I $\pm 0.036, n=23$ slices (2 animals); $t_{(54)}=3.61$, $p=0.00068, r=0.44$ ). (b) Z-stacked images at $\times 40$ magnification Zoom $2 x$ in I00- $\mu$ m-thick slice (scale bar $=25 \mu \mathrm{m}$ ), the first column (left) in green shows $\mathrm{CBIR}$ labeling, second column (middle) in white shows NeuN, and column 3 (right) shows the merge of signals. (c) Representative $\times 10$ magnification Z-stacked images taken from dorsal hippocampal sections (scale bar $=200 \mu \mathrm{m}$ ). (d) Mean intensity of the CBIR fluorescent signal (normalized to NeuN signal taken from pyramidal cell layer) in the CAI region of the dorsal hippocampus shows no differences between treatment groups (PCP treated: $1.03 \pm 0.017, n=28$ slices (4 animals); CTRL: I $\pm 0.022, n=22$ slices ( 3 animals); $t_{(48)}=0.91 \mathrm{I}, p=0.3667, r=0.0 .13$ ). (e) Representative $\times 40$ magnification images taken from the CAI region used for analysis and quantification. Normalization to NeuN was taken from regions of interest (ROIs) $\mathrm{I}$ and 2 (pyramidal cell layer), and level of CBIR expression was taken from all ROls combined. ROls extended down into the striatum radiatum with an additional two rows (ROIs 5, 6, 7, and 8 not shown) (scale bar $=50 \mu \mathrm{m}$ ). (f) Level of CBIR in dentate gyrus (DG) was slightly increased (PCP treated: I. $12 \pm 0.027, n=28$ slices (4 animals); CTRL: I $\pm 0.015, n=22$ slices ( 3 animals); $t_{(50)}=3.65, p=0.0006, r=0.46$ ). (g) Representative $\times 40$ magnification images taken from the DG region used for analysis and quantification. Normalization to NeuN was taken from ROls I and 2 of the granule cell layer, and the level of CBIR expression was taken from all ROls combined $(I-4)$ (scale bar $=50 \mu \mathrm{m}$ ). A full color version of this figure is available at the Neuropsychopharmacology journal online. Asterisks indicate statistical significance, $* p<0.05$, $* * 0<0.01$, $* * * * 0.001$.

signaling in PCP-treated mice. However, we have not found any abnormality in functionality of CB1R-dependent signaling at the $\mathrm{CA} 3 \rightarrow \mathrm{CA} 1$ synapse in the hippocampus in PCP-treated mice (Figure 5e; RM-ANOVA: Time $\times$ Treatment: $\left.\mathrm{F}_{(2.0,20.0)}=1.077, p=0.384\right)$. In addition, the total levels of CB1R in CA1 (including CA3 $\rightarrow$ CA1 synapses) 

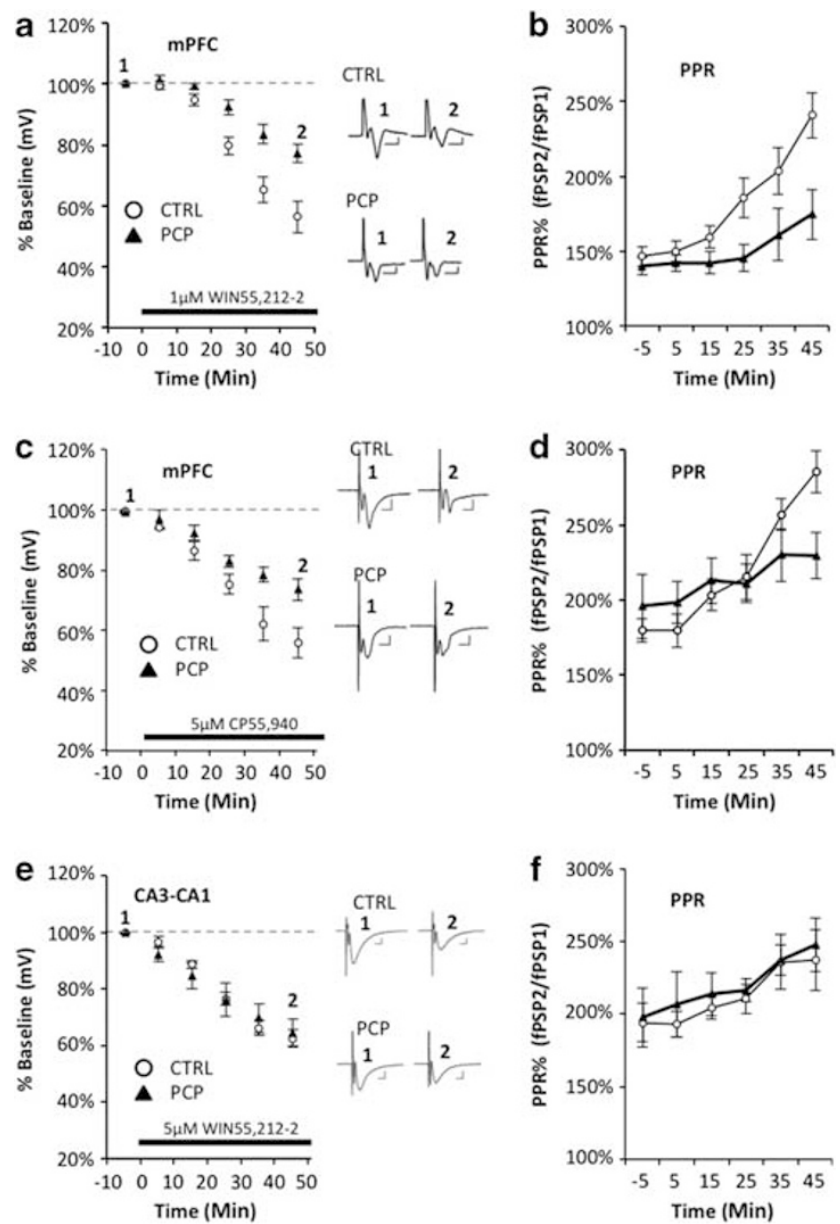

Figure 5 Cannabinoid receptor type I (CB|R) function is reduced in phencyclidine (PCP)-treated mice. (a) The PCP-treated group shows decreased functionality of $C B I R$ at the layer $(L) 2 / 3 \rightarrow L 5$ synapse in the medial prefrontal cortex (mPFC) when compared with control (CTRL) mice. After 10 min of baseline recording, continuous application of I $\mu \mathrm{M}$ WIN55 2I 2-2 (CBIR agonist) caused a reduction of field postsynaptic potential (PPS) amplitude in the CTRL group, which was markedly dampened in the PCP-treated mice. Insets: Traces shown indicate representative average from 10 min of baseline (' $\mathrm{I}$ ') and the time between 40 and 50 min of recording (' 2 ') (scale bar: vertical $=0.1 \mathrm{mV}$; horizontal $=5$ $m s)$. ( $b, d$, and f) Paired-pulse ratios (PPRs) were recorded periodically over the course of drug application. Decreases in fPSP (a) correlate well with PPRs in CTRL and PCP-treated mice over time. This increase in PPR is consistent with a presynaptic locus of action. (c) Continuous application of $5 \mu$ M CP55 940 (CBIR agonist) caused a reduction of fPSP amplitude in the CTRL group, which was also markedly dampened in the PCP-treated mice (repeated measures-ANOVA (RM-ANOVA): Time $\times$ Treatment: $\left.F_{(2.1,31.4)}=3.797, p=0.032\right)$. (e) The PCP-treated group shows no difference in functionality of $C B I R$ at the $C A 3 \rightarrow C A I$ synapse when compared with CTRL mice. Continuous application of $5 \mu$ M WIN55 212-2 (CBIR agonist) caused a similar reduction of fPSP amplitude in both groups of mice (RM-ANOVA: Time $\times$ Treatment: $\left.F_{(2.0,20.0)}=1.077, p=0.384\right)$.

within the hippocampus did not differ between PCP-treated and control animals (Figures $4 \mathrm{~d}$ and e) (PCP treated: $1.03 \pm 0.017, n=28$; CTRL: $1 \pm 0.022, n=22 ; t_{(48)}=0.911$, $p=0.3667, r=0.013$ ), while in the DG region (including cortical projections from entorhinal cortex) they were slightly increased in PCP-treated mice (Figures $4 \mathrm{f}$ and $\mathrm{g}$ ). These data suggest that the disruption in the eCB signaling observed in PCP mice is not widespread.

\section{DISCUSSION}

NMDAR hypofunction during development is known to produce structural and functional abnormalities of cortical networks in the adult brain, although the neural mechanisms of emergent pathology and its consequences on cognitive function are unclear. It has been shown that PCP-mediated NMDAR hypofunction during early postnatal development before PND 12 results in cognitive dysfunction, including working memory deficits (Nakatani-Pawlak et al, 2009) and object recognition deficits (Grayson et al, 2007; NakataniPawlak et al, 2009; Wiley et al, 2003) in rodent models of psychosis. Our data strongly suggested that transient PCPmediated NMDAR hypofunction during early postnatal development (PND 7-11) resulted in imbalanced fear memory specificity and generalization in the adult.

Theoretical and experimental models predict coexistence of two anatomically distinctive neural processes underlying specificity of episodic memory (Leutgeb et al, 2007; Marr, 1971; McHugh et al, 2007; O'Reilly and McClelland, 1994). Pattern separation is a putative neural process underlying memory specificity, in which memories are encoded and retrieved as unique representations, while pattern completion enables recall based on partial cues and memory generalization. It is uncertain if the abnormality in context discrimination found in PCP-mediated NMDAR hypofunction resulted from overgeneralization or a deficit in pattern separation. The possibility for a disruption of hippocampusdependent pattern separation/completion in schizophrenia has been discussed previously (Tamminga et al, 2010). However, the neural circuit for memory specificity and generalization also involves the PFC and thalamus (Navawongse and Eichenbaum, 2013; Xu et al, 2012; Xu and Sudhof, 2013), and there is converging evidence linking the schizophrenia cognitive endophenotype with NMDAR hypofunction-induced abnormalities in the thalamus, PFC and hippocampus circuit, consistent with the NMDAR hypofunction theory (Coyle et al, 2003; Tamminga, 1998). We suggest that impairment in fear memory specificity observed in adult animals may be associated with an abnormal maturation of plasticity as revealed in PCP-mediated NMDAR hypofunction mice.

PCP-treated mice exhibited a loss of eCB-dependent synaptic plasticity at a central glutamatergic synapse, suggesting that abnormality in fear memory specificity and generalization may be associated with deficient eCBdependent plasticity. Recent studies indicate that schizophrenia pathology may be linked to the eCB system (Caspi et al, 2005; Zammit et al, 2011). Furthermore, mutations in CNR1 (a gene encoding CB1R) have been associated with a susceptibility to schizophrenia in human genetic studies (Leroy et al, 2001; Ujike et al, 2002). Studies of mutant mice with the CNR1 gene deletion revealed that the schizophrenia-like behavioral responses to an NMDAR inhibitor depended on a functional CB1R (Haller et al, 2005). Thus, our data indicating an abnormality in the $\mathrm{eCB}$ system in a schizophrenia model are consistent with the initial findings in humans and rodents.

Our initial observation indicated a loss of eCB-LTD (Figure 3b), which coincides with a decrease in CB1R function in PCP-treated mice (Figure 5). However, this modest decrease in $\mathrm{CB} 1 \mathrm{R}$ function may be insufficient to explain the loss of eCB-LTD induction, and it is uncertain at 
this point if additional components of the eCB system are dysregulated in PCP-treated mice. For example, either decreased $\mathrm{eCB}$ synthesis or increased $\mathrm{eCB}$ degradation will attenuate eCB-LTD. This might occur if PCP-treatment leads to enduring changes in expression of the enzymes mediating eCB synthesis and degradation (see below). eCBLTD was first discovered in the glutamatergic striatal synapse (Calabresi et al, 1992), although the role of the $\mathrm{eCB}$ system including CB1R in this form of plasticity was not known until later (Gerdeman et al, 2002). At the L2/3 $\rightarrow$ L5 synapse in the $\mathrm{mPFC}$, the retrograde eCB signaling system depends on complex presynaptic and postsynaptic signaling (Lovinger, 2008) through the CB1R (Lafourcade et al, 2007), metabotropic glutamate receptor (mGluR5, that transduces the level of glutamate onto the postsynaptic compartment) (Calabresi et al, 1992; Lafourcade et al, 2007), diacylglycerol lipase (DAGL $\alpha$ is essential for the eCB 2-arachidonoylglycerol (2-AG) synthesis) (Lafourcade et al, 2007; Lovinger, 2008), $\mathrm{Ca}^{2+}$ influx (Di Marzo et al, 1994), and MAGL and $\alpha / \beta$-hydrolase domain 6 (MAGL and ABHD6, respectively, enzymes that degrade 2-AG released from the postsynaptic terminal) (Marrs et al, 2010; Piomelli, 2003). The eCB-LTD mechanism may also involve serotonin receptors (5-HT2) (Best and Regehr, 2008), dopamine receptors (D2) (Calabresi et al, 1992), or muscarinic acetylcholine receptors (M1/3) (Kim et al, 2002). In addition, eCB-LTD is a widespread type of long-term synaptic plasticity found at excitatory and some inhibitory synapses in the CNS (Lovinger, 2008). The extent to which the eCB system is disrupted in PCP-treated mice has yet to be determined, but it is unlikely that this form of synaptic plasticity is diffusely deficient throughout the brain.

The induced NMDAR hypofunction in PCP-treated mice is overlapping at a time when the intrinsic characteristics of the pyramidal neurons in L5 and the properties of L2/3 inputs in the $\mathrm{MPFC}$ are developing to form mature plasticity mechanisms at this glutamatergic pathway (Zhang, 2004). Our data indicate that NMDARs are required for the maturation of eCB-dependent presynaptic plasticity at the glutamatergic synapse in addition to the well-studied maturation of postsynaptic plasticity.

The retrograde eCB inhibitory system demonstrates a number of properties that make it a viable candidate for a homeostatic mechanism controlling neural stability that can be permanently disrupted via adaptive mechanisms. There are two critical characteristics that make the eCB system an effective component of the neuronal activity-dependent homeostatic mechanism. First, eCBs are produced locally and postsynaptically. Second, the retrograde release of eCBs exhibits temporal and spatial patterns that faithfully follow the patterns of neuronal activity at presynaptic terminals (Lovinger, 2008). However, recent studies have indicated that the $\mathrm{eCB}$ system is vulnerable to disruption via maladaptive mechanisms induced through environmental insults. Both epilepsy and chronic exposure to a CB1R antagonist induce long-lasting changes in the expression of CB1R (Chen et al, 2003; Hsieh et al, 1999; Rinaldi-Carmona et al, 1998). Repeated self-administered cocaine resulted in an impairment of two forms of long-term plasticity, such as eCB-LTD and mGlu2/3-mediated LTD in the mPFC (Kasanetz et al, 2012). The loss of eCB-LTD was also observed in response to a single exposure to $\Delta^{9}$-tetrahy- drocannabinol in the nucleus accumbens as a result of functional tolerance and the desensitization of CB1R (Mato et al, 2004). However, prolonged exposure to this drug induces compensatory mechanisms and rescues LTD via mGlu2/3-dependent homeostatic synaptic adaptation (Mato et al, 2005). Excitatory synapses onto indirect-pathway medium spiny neurons selectively express dopamine D2 receptor-dependent eCB-LTD, which is absent in Parkinson's disease models, and can be rescued through the pharmacological restoration of eCB-LTD expression (Calabresi et al, 1992; Gerdeman et al, 2002; Kreitzer and Malenka, 2007). Thus, long-term synaptic depression, including eCB-LTD, is involved in cortical and subcortical maladaptations, and our current data suggest that the eCBLTD mechanism might be a target relevant to the developmental etiology underlying neuropsychiatric disorders. However, the specific roles of disrupted eCB-LTD on network stability, synaptic plasticity, and cognitive function have yet to be determined.

\section{FUNDING AND DISCLOSURE}

This study was supported by the NIH/NIMH grant MH086078 (to EK), NIH/NIDA grants DA011322 and DA021696 (to KM), and Ford Fellowship (to PAV). The authors declare no conflict of interest.

\section{ACKNOWLEDGEMENTS}

We thank Alia Bana, Alex Hiroto and Christopher L Hughes for their technical assistance; and David $M$ Lovinger and John Lisman for comments on the manuscript.

\section{REFERENCES}

Best AR, Regehr WG (2008). Serotonin evokes endocannabinoid release and retrogradely suppresses excitatory synapses. J Neurosci 28: 6508-6515.

Calabresi P, Maj R, Pisani A, Mercuri NB, Bernardi G (1992). Longterm synaptic depression in the striatum: physiological and pharmacological characterization. J Neurosci 12: 4224-4233.

Caspi A, Moffitt TE, Cannon M, McClay J, Murray R, Harrington H et al (2005). Moderation of the effect of adolescent-onset cannabis use on adult psychosis by a functional polymorphism in the catechol-O-methyltransferase gene: longitudinal evidence of a gene $\times$ environment interaction. Biol Psychiatry 57: 1117-1127.

Chen K, Ratzliff A, Hilgenberg L, Gulyas A, Freund TF, Smith M et al (2003). Long-term plasticity of endocannabinoid signaling induced by developmental febrile seizures. Neuron 39: 599-611.

Coyle JT, Tsai G, Goff D (2003). Converging evidence of NMDA receptor hypofunction in the pathophysiology of schizophrenia. Ann N Y Acad Sci 1003: 318-327.

Di Marzo V, Fontana A, Cadas H, Schinelli S, Cimino G, Schwartz JC et al (1994). Formation and inactivation of endogenous cannabinoid anandamide in central neurons. Nature 372: 686-691.

Gerdeman GL, Ronesi J, Lovinger DM (2002). Postsynaptic endocannabinoid release is critical to long-term depression in the striatum. Nat Neurosci 5: 446-451.

Grayson B, Idris NF, Neill JC (2007). Atypical antipsychotics attenuate a sub-chronic PCP-induced cognitive deficit in the novel object recognition task in the rat. Behav Brain Res 184: 31-38.

Haller J, Szirmai M, Varga B, Ledent C, Freund TF (2005). Cannabinoid CB1 receptor dependent effects of the NMDA 
antagonist phencyclidine in the social withdrawal model of schizophrenia. Behav Pharmacol 16: 415-422.

Hempel CM, Hartman KH, Wang XJ, Turrigiano GG, Nelson SB (2000). Multiple forms of short-term plasticity at excitatory synapses in rat medial prefrontal cortex. J Neurophysiol 83: 3031-3041.

Hsieh C, Brown S, Derleth C, Mackie K (1999). Internalization and recycling of the CB1 cannabinoid receptor. J Neurochem 73: 493-501.

Javitt DC, Zukin SR (1991). Recent advances in the phencyclidine model of schizophrenia. Am J Psychiatry 148: 1301-1308.

Kasanetz F, Lafourcade M, Deroche-Gamonet V, Revest JM, Berson $\mathrm{N}$, Balado E et al (2012). Prefrontal synaptic markers of cocaine addiction-like behavior in rats. Mol Psychiatry 18: 729-737.

Kim J, Isokawa M, Ledent C, Alger BE (2002). Activation of muscarinic acetylcholine receptors enhances the release of endogenous cannabinoids in the hippocampus. J Neurosci 22: 10182-10191.

Korzus E, Rosenfeld MG, Mayford M (2004). CBP histone acetyltransferase activity is a critical component of memory consolidation. Neuron 42: 961-972.

Kreitzer AC, Malenka RC (2007). Endocannabinoid-mediated rescue of striatal LTD and motor deficits in Parkinson's disease models. Nature 445: 643-647.

Lafourcade M, Elezgarai I, Mato S, Bakiri Y, Grandes P, Manzoni OJ (2007). Molecular components and functions of the endocannabinoid system in mouse prefrontal cortex. PLoS One 2: e709.

Leroy S, Griffon N, Bourdel MC, Olie JP, Poirier MF, Krebs MO (2001). Schizophrenia and the cannabinoid receptor type 1 (CB1): association study using a single-base polymorphism in coding exon 1. Am J Med Genet 105: 749-752.

Leutgeb JK, Leutgeb S, Moser MB, Moser EI (2007). Pattern separation in the dentate gyrus and CA3 of the hippocampus. Science 315: 961-966.

Long JZ, Li W, Booker L, Burston JJ, Kinsey SG, Schlosburg JE et al (2009). Selective blockade of 2-arachidonoylglycerol hydrolysis produces cannabinoid behavioral effects. Nat Chem Biol 5: 37-44.

Lovinger DM (2008). Presynaptic modulation by endocannabinoids. Handb Exp Pharmacol 184: 435-477.

Makara JK, Mor M, Fegley D, Szabo SI, Kathuria S, Astarita G et al (2005). Selective inhibition of 2-AG hydrolysis enhances endocannabinoid signaling in hippocampus. Nat Neurosci 8: 1139-1141.

Marr D (1971). Simple memory: a theory for archicortex. Philos Trans R Soc Lond Ser B 262: 23-81.

Marrs WR, Blankman JL, Horne EA, Thomazeau A, Lin YH, Coy J et al (2010). The serine hydrolase ABHD6 controls the accumulation and efficacy of 2-AG at cannabinoid receptors. Nat Neurosci 13: 951-957.

Mato S, Chevaleyre V, Robbe D, Pazos A, Castillo PE, Manzoni OJ (2004). A single in-vivo exposure to delta 9THC blocks endocannabinoid-mediated synaptic plasticity. Nat Neurosci 7: 585-586.

Mato S, Robbe D, Puente N, Grandes P, Manzoni OJ (2005). Presynaptic homeostatic plasticity rescues long-term depression after chronic Delta 9-tetrahydrocannabinol exposure. J Neurosci 25: 11619-11627.

McHugh TJ, Jones MW, Quinn JJ, Balthasar N, Coppari R, Elmquist JK et al (2007). Dentate gyrus NMDA receptors mediate rapid pattern separation in the hippocampal network. Science 317: 94-99.

Morris BJ, Cochran SM, Pratt JA (2005). PCP: from pharmacology to modelling schizophrenia. Curr Opin Pharmacol 5: 101-106.

Morris SH, Knevett S, Lerner EG, Bindman LJ (1999). Group I mGluR agonist DHPG facilitates the induction of LTP in rat prelimbic cortex in vitro. J Neurophysiol 82: 1927-1933.
Nakatani-Pawlak A, Yamaguchi K, Tatsumi Y, Mizoguchi H, Yoneda Y (2009). Neonatal phencyclidine treatment in mice induces behavioral, histological and neurochemical abnormalities in adulthood. Biol Pharm Bull 32: 1576-1583.

Navawongse R, Eichenbaum H (2013). Distinct pathways for rulebased retrieval and spatial mapping of memory representations in hippocampal neurons. J Neurosci 33: 1002-1013.

O'Reilly RC, McClelland JL (1994). Hippocampal conjunctive encoding, storage, and recall: avoiding a trade-off. Hippocampus 4: 661-682.

Pelletier M, Achim AM, Montoya A, Lal S, Lepage M (2005). Cognitive and clinical moderators of recognition memory in schizophrenia: a meta-analysis. Schizophr Res 74: 233-252.

Piomelli D (2003). The molecular logic of endocannabinoid signalling. Nat Rev Neurosci 4: 873-884.

Reichenberg A, Caspi A, Harrington H, Houts R, Keefe RS, Murray RM et al (2010). Static and dynamic cognitive deficits in childhood preceding adult schizophrenia: a 30-year study. Am J Psychiatry 167: 160-169.

Rinaldi-Carmona M, Le Duigou A, Oustric D, Barth F, Bouaboula M, Carayon P et al (1998). Modulation of CB1 cannabinoid receptor functions after a long-term exposure to agonist or inverse agonist in the Chinese hamster ovary cell expression system. J Pharmacol Exp Ther 287: 1038-1047.

Robbe D, Alonso G, Chaumont S, Bockaert J, Manzoni OJ (2002). Role of $\mathrm{p} / \mathrm{q}-\mathrm{Ca}^{2}+$ channels in metabotropic glutamate receptor 2/3-dependent presynaptic long-term depression at nucleus accumbens synapses. J Neurosci 22: 4346-4356.

Sahay A, Wilson DA, Hen R (2011). Pattern separation: a common function for new neurons in hippocampus and olfactory bulb. Neuron 70: $582-588$.

Tamminga CA (1998). Schizophrenia and glutamatergic transmission. Crit Rev Neurobiol 12: 21-36.

Tamminga CA (2013). Psychosis is emerging as a learning and memory disorder. Neuropsychopharmacology 38: 247.

Tamminga CA, Stan AD, Wagner AD (2010). The hippocampal formation in schizophrenia. Am J Psychiatry 167: 1178-1193.

Tulving E (2002). Episodic memory: from mind to brain. Annu Rev Psychol 53: 1-25.

Ujike H, Takaki M, Nakata K, Tanaka Y, Takeda T, Kodama M et al (2002). CNR1, central cannabinoid receptor gene, associated with susceptibility to hebephrenic schizophrenia. Mol Psychiatry 7: 515-518.

Wang C, McInnis J, Ross-Sanchez M, Shinnick-Gallagher P, Wiley JL, Johnson KM (2001). Long-term behavioral and neurodegenerative effects of perinatal phencyclidine administration: implications for schizophrenia. Neuroscience 107: 535-550.

Wiley JL, Buhler KG, Lavecchia KL, Johnson KM (2003). Pharmacological challenge reveals long-term effects of perinatal phencyclidine on delayed spatial alternation in rats. Prog Neuropsychopharmacol Biol Psychiatry 27: 867-873.

Xu W, Morishita W, Buckmaster PS, Pang ZP, Malenka RC, Sudhof TC (2012). Distinct neuronal coding schemes in memory revealed by selective erasure of fast synchronous synaptic transmission. Neuron 73: 990-1001.

Xu W, Sudhof TC (2013). A neural circuit for memory specificity and generalization. Science 339: 1290-1295.

Zammit S, Owen MJ, Evans J, Heron J, Lewis G (2011). Cannabis, COMT and psychotic experiences. Br J Psychiatry 199: 380-385.

Zhang ZW (2004). Maturation of layer V pyramidal neurons in the rat prefrontal cortex: intrinsic properties and synaptic function. J Neurophysiol 91: 1171-1182.

Supplementary Information accompanies the paper on the Neuropsychopharmacology website (http://www.nature.com/npp) 